\title{
The Role of Self-Objectification in the Experience of Women with Eating Disorders
}

\author{
Rachel M. Calogero, ${ }^{1,3}$ William N. Davis, ${ }^{1}$ and J. Kevin Thompson ${ }^{2}$
}

\begin{abstract}
Objectification theory has linked self-objectification to negative emotional experiences and disordered eating behavior in cultures that sexually objectify the female body. This link has not been empirically tested in a clinical sample of women with eating disorders. In the present effort, 209 women in residential treatment for eating disorders completed self-report measures of self-objectification, body shame, media influence, and drive for thinness on admission to treatment. Results demonstrated that the internalization of appearance ideals from the media predicted self-objectification, whereas using the media as an informational source about appearance and feeling pressured to conform to media ideals did not. Selfobjectification partially mediated the relationship between internalized appearance ideals and drive for thinness; internalized appearance ideals continued to be an independent predictor of variance. In accordance with objectification theory, body shame partially mediated the relationship between self-objectification and drive for thinness in women with eating disorders; self-objectification continued to be an independent predictor of variance. These results illustrate the importance of understanding and targeting the experience of self-objectification in women with eating disorders or women at risk for eating disorders.
\end{abstract}

KEY WORDS: self-objectification; eating disorders; internalization; sociocultural.

Sexual objectification occurs whenever a person is viewed, evaluated, reduced to, and/or treated by others as merely a body, and has been identified as especially harmful to women (Bartky, 1990; Fredrickson \& Roberts, 1997; Kaschak, 1992). Objectification theory considers self-objectification to be an adaptive strategy for girls and women who live in a culture that sexually objectifies the female body (Fredrickson \& Roberts, 1997). The term selfobjectification refers to the psychological process by which women internalize observers' objectifying perspectives on their bodies and become chronic selfmonitors of their own physical appearance. This form of self-perception allows women to anticipate, and

\footnotetext{
${ }^{1}$ Department of Psychology, The Renfrew Center Foundation, Syracuse University, Syracuse, New York.

${ }^{2}$ University of South Florida, Tampa, Florida.

${ }^{3}$ To whom correspondence should be addressed at Department of Psychology, Syracuse University, 430 Huntington Hall, Syracuse, New York 13244; e-mail: rmcaloge@syr.edu.
}

thus to exert some control over, how others treat them.

By coming to view their own bodies through this objectified lens, women engage in a kind of psychologically distancing from their physical bodies, which in turn contributes to negative attitudes toward and experiences with their bodies. This experience of self-objectification has been theoretically and empirically linked to a variety of negative consequences in nonclinical samples of adult and adolescent women, including increased opportunities for experiencing body shame, anxiety, negative attitudes toward menstruation, a disrupted flow of consciousness, diminished awareness of internal bodily states, depression, sexual dysfunction, and disordered eating (Calogero, 2004; Fredrickson, Roberts, Noll, Quinn, \& Twenge, 1998; Noll \& Fredrickson, 1998; Roberts, 2004; Slater \& Tiggemann, 2002; Tiggemann \& Slater, 2001).

Self-objectification may be a critical factor to identify for the treatment and recovery of women 
with eating disorders. This form of self-perception has not been examined empirically in a clinically eating disordered population, where disconnection and distancing from the body is prominent. Eating disorders have been described, in part, as women's response to feeling powerless to control the systematic objectification of their bodies (Fredrickson \& Roberts, 1997). An exploration of the role of selfobjectification in this population of women may provide information about the cultural construction of eating disorders that extends beyond fat phobia to include broader sociocultural forces.

To begin, it is necessary to consider what specific factors might contribute to the experience of self-objectification in women with eating disorders. Fredrickson and Roberts (1997) theorized that the virtually unavoidable exposure to sexualized depictions of female bodies and body parts in the visual media is a major contributor to this form of self-perception. Recently, researchers have demonstrated that reading contemporary woman's magazines for appearance-based and gender role advice was positively related to objectifying one's own body and accepting the content of the messages (Kim \& Ward, 2004). A meta-analytic review of 43 tests of the immediate impact of exposure to the thin beauty ideal produced a small but consistent and significant effect size (Groesz, Levine, \& Murnen, 2002), which demonstrates that the visual media also contribute to experiences of negative body image. Based on a review of various research designs and methodologies, Stice (2001) has suggested that the negative effects of media exposure could be considered a causal risk factor for eating disturbances.

Several dimensions of the media's potential influence on women's beliefs about and behavior toward their bodies have been examined recently in nonclinical and clinical eating disordered samples (Calogero, Davis, \& Thompson, 2004; Thompson, van den Berg, Roehrig, Guarda, \& Heinberg, 2004). These dimensions include the degree to which media are considered important sources of information about appearance, feeling pressured by media to conform to ideal standards, and internalizing general and athletic body ideals. A clinical sample of 440 eating disordered inpatients scored higher across almost all dimensions of media influence than did a nonclinical sample of 380 college students. The one exception was the dimension that measured the importance of the media as an informational source about appearance, where the nonclinical sample was slightly higher or roughly equivalent to the clinical sample.
Thus, the first purpose of the present study was to examine which dimensions of media influence, if any, predict self-objectification among women in a residential eating disordered population. The second purpose was to examine whether there was a direct or indirect relationship between dimensions of media influence and eating disorder pathology, with selfobjectification as the hypothesized mediator, among women in a residential eating disordered population. A cardinal feature of eating disorders is some degree of caloric restriction and excessive concern with weight (American Psychiatric Association, 1994; Bruch, 1973, 1982). To approximate the assessment of this feature of eating disorders pathology, a measure of drive for thinness was utilized.

Next, the contribution of self-objectification itself to experiences of body shame and disordered eating in women with eating disorders was considered. Recurrent opportunities for women to experience body shame in both public and private contexts are powerful motivators to engage in disordered eating. Noll and Fredrickson (1998) demonstrated that body shame mediated the relationship between self-objectification and disordered eating behaviors in nonclinical samples, and they found that both predictors contributed independently to the variance in disordered eating. Similarly, women with eating disorders may behave in unhealthy ways in an attempt to alleviate feelings of shame about the body or, by self-objectifying, to avoid the experience of shame altogether. Thus, the final purpose of the present study was to examine whether there is a direct or indirect relationship between self-objectification and eating disorder pathology, with body shame as the hypothesized mediator, among women in residential treatment for eating disorders. Again, drive for thinness was utilized as the measure of eating disorders pathology in this model.

\section{METHOD}

\section{Participants}

Participants were 209 women in a residential treatment facility for women with eating disorders. Only patients who provided research consent upon admission were included in the present study. Patients were diagnosed on the basis of a semi-structured interview by a senior staff psychiatrist using criteria from the Diagnostic and Statistical Manual of Mental Disorders (4th ed., 
American Psychiatric Association, 1994). In this sample, $31.2 \%$ were diagnosed with anorexia nervosarestricting subtype (ANR; $n=69$ ), $13.7 \%$ with anorexia nervosa-binge-purge subtype (ANBP; $n=$ $27), 35 \%$ with bulimia nervosa ( $\mathrm{BN} ; n=70)$, and $20.2 \%$ with eating disorder not otherwise specified (EDNOS; $n=43$ ). Mean body mass index (BMI) was 19.70 , and mean age was 22.90 years. Ninetyfive percent of the women were European American, $1.8 \%$ were African American, $1.8 \%$ Asian, and $1.4 \%$ marked unspecified "other."

\section{Measures}

\section{Self-Objectification Questionnaire}

The Self-Objectification Questionnaire (SOQ; Fredrickson et al., 1998; Noll \& Fredrickson, 1998) assesses the extent to which individuals view their bodies in observable, appearance-based terms versus nonobservable, competence-based terms without an evaluation of how satisfied they are with their bodies. Participants rank ordered 10 body attributes by how important each was to their own physical self-concept from 0 (least impact) to 9 (greatest im$p a c t$ ). Difference scores were computed by subtracting the sum of the 5 competence attributes (e.g., health, strength) from the sum of the 5 appearance attributes (e.g., physical attractiveness, weight). Scores ranged from -25 to 25 ; higher scores indicate greater self-objectification. Consistent with objectification theory, self-objectification scores were uncorrelated with BMI in this sample $(r=.05, n s)$. Satisfactory construct validity has been shown elsewhere (Noll \& Fredrickson, 1998).

\section{Body Shame Questionnaire}

The Body Shame Questionnaire (Fredrickson et al., 1998) assesses the phenomenological aspects of body shame that occur when individuals imagine looking at themselves in the mirror. This indirect measure of shame circumvents the difficulty of eliciting accurate responses from individuals who may feel ashamed of being ashamed. Participants rated 18 items comprising motivational and behavioral components of shame (e.g., "I wish I were invisible," "I feel like crawling into a corner") using a 5-point Likert scale from 1 (not at all) to 5 (extremely). Scores ranged from 18 to 90; higher scores indicate greater body shame when looking at oneself in the mirror. Cronbach's alpha indicated high internal consistency across the 18 items $(\alpha=.95)$.

\section{Sociocultural Attitudes Toward Appearance Scale-3}

The Sociocultural Attitudes Toward Appearance Scale-3 (SATAQ-3; Thompson et al., 2004) is a 30-item questionnaire that assesses four dimensions of media influence on body image and eating disturbances: information, pressures, internalizationgeneral, and internalization-athletic. The information dimension includes 9 items that measure the degree to which various media are important sources for obtaining information about attractiveness and fashion (e.g., "Magazine articles are an important source of information about fashion and being attractive"). The pressures dimension includes 7 items that measure the degree to which individuals have felt pressured by various media to change their appearance (e.g., "I've felt pressure from TV and magazines to lose weight"). The general internalization dimension includes 9 items that measure the degree to which individuals have "bought into" the norms for body shape and weight portrayed in the media and the degree to which they attempt to match these standards by modifying certain behaviors (e.g., "I would like my body to look like the people who are on TV"). The athletic internalization dimension includes 5 items that measure the degree to which individuals desire and strive to achieve the bodies of athletes portrayed in the media (e.g., "I wished I looked as athletic as sports stars"). High internal consistency has been shown for the 4 dimensions in nonclinical ( $\alpha=.96, .92, .95$, and .96 , respectively) and eating disordered populations $(\alpha=.94-.96, .94-$ $.95, .93-.97$, and $.77-.84$, respectively) as well as good construct validity (Calogero et al., 2004; Thompson et al., 2004). In the present study, the general and athletic internalization dimensions were combined for use in model testing given that the underlying construct of internalization is the same and to simplify interpretation of the data. Cronbach's alphas indicated high internal consistency for the information, pressures, and internalization dimensions examined in this study ( $\alpha=.95, .96$, and .86 , respectively).

\section{Drive for Thinness Subscale}

The Eating Disorder Inventory-Drive for Thinness subscale (EDI—DT; Garner, 1991) assesses 
excessive concern with dieting, fear of weight gain, and preoccupation with a desire to be thinner. Excellent reliability and validity has been demonstrated in samples of eating disordered patients (Garner, 1991; Garner, Olmstead, \& Polivy, 1983). In the present study, Cronbach's alpha indicated high internal consistency $(\alpha=.94)$.

\section{Procedure}

Women were administered the packet of questionnaires in counterbalanced order on admission to residential treatment. All of the assessment information was administered and collected in a sealed packet to maintain patient confidentiality. Informed consent was obtained prior to completion of any questionnaires.

\section{RESULTS}

Means, standard deviations, and the range of scores for self-objectification, body shame, dimensions of media influence, and drive for thinness are shown in Table I. The zero-order correlations among them are displayed in Table II. The Information dimension of the SATAQ-3 was the only variable not significantly correlated with drive for thinness. Positive correlations among self-objectification, body shame, and drive for thinness replicate previous findings with nonclinical samples (Fredrickson et al., 1998; Noll \& Fredrickson, 1998). The mean self-objectification score in the current sample should be noted. Compared to nonclinical samples, the current mean self-objectification score of 9.05 is remarkably higher than the mean self-objectification

Table I. Means and Variability for Measures at Admission to Treatment

\begin{tabular}{|c|c|c|c|}
\hline Measure & $\mathrm{M}(S D)$ & Minimum & Maximum \\
\hline Self-objectification & $9.05(13.53)$ & -25.00 & 25.00 \\
\hline Body shame & $63.94(19.77)$ & 23.00 & 90.00 \\
\hline Drive for thinness & $24.08(12.42)$ & 0.00 & 42.00 \\
\hline \multicolumn{4}{|l|}{ Media influence } \\
\hline Information & $29.90(9.20)$ & 9.00 & 45.00 \\
\hline Pressures & $26.15(7.70)$ & 7.00 & 35.00 \\
\hline Internalization-general $^{a}$ & $34.48(9.26)$ & 9.00 & 45.00 \\
\hline Internalization-athlete & $18.36(4.07)$ & 5.00 & 25.00 \\
\hline
\end{tabular}

Note. $n=209$.

${ }^{a}$ Internalization— general and athlete were combined to produce one internalization score prior to model testing.
Table II. Correlations Among Measures

\begin{tabular}{lcccccc}
\hline Measure & BS & Info & Press & Int-G & Int-A & DT \\
\hline SO & .32 & .29 & .34 & .49 & .35 & .38 \\
BS & - & .28 & .28 & .36 & .39 & .44 \\
Info & - & - & .55 & .55 & .41 & .17 \\
Press & - & - & - & .78 & .48 & .28 \\
Int-G & - & - & - & - & .66 & .38 \\
Int-A & - & - & - & - & - & .39 \\
\hline
\end{tabular}

Note. All correlations are significant at $p<.01$ except information/drive for thinness $(n s) . n=209$; SO = self-objectification; $\mathrm{BS}=$ body shame; Info = information; Press = pressures; Int- $\mathrm{G}=$ internalization—general; Int-A = internalization—athlete; DT = drive for thinness.

scores previously reported, which have ranged between -10.34 and 7.70 (e.g., Fredrickson et al., 1998; Noll \& Fredrickson, 1998; Tiggeman \& Lynch, 2001; Tiggemann \& Slater, 2001).

\section{Media Influence and Self-Objectification}

To test the relationship between media influence and self-objectification, the three dimensions of media influence measured by the SATAQ-3 were entered hierarchically into a regression model with information first, pressures second, and internalization third, based on their theoretical temporal order. When self-objectification was regressed on the SATAQ-3, Information, $\beta=.08, t(206)=1.09, p=$ .28 , and Pressures, $\beta=-.003, t(206)=-0.03, p=$ .24 , from media were not significant predictors of self-objectification. Internalization of media was the only significant predictor; it accounted for $8 \%$ of the variance in self-objectification, $\beta=.41, t(206)=$ $4.56, p<.0001$, and the entire model explained $21 \%$ of the variance, $F(3,206)=17.14, p<.0001$.

\section{Media Influence, Self-Objectification, and Drive for Thinness}

The two mediational models of eating disorder pathology were tested using multiple regression analyses. According to Baron and Kenny (1986), 3 steps are necessary to establish mediation. In the first step, the predictor variable must correlate with the outcome variable. In the second step, the predictor variable must correlate with the hypothesized mediator. In the third step, when the predictor and mediator are entered simultaneously into the regression equation, the mediator must correlate with the outcome 


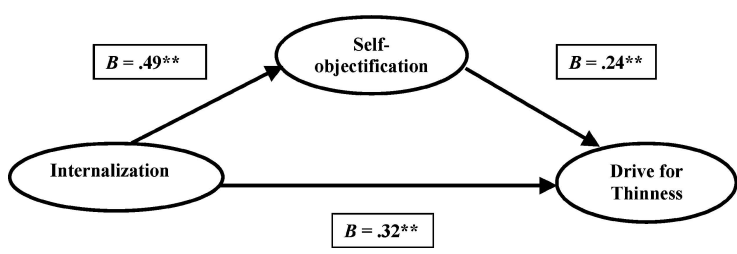

Fig. 1. Beta coefficients for the pathways among internalization, self-objectification, and drive for thinness $\left({ }^{* *} p<.001\right)$.

variable and the relationship between the predictor and the outcome should weaken.

Because internalization was the only significant predictor of self-objectification, it was the only factor of the SATAQ-3 included in the test of the first mediational model. Figure 1 displays the results of a series of regression equations computed to test this model. In the first equation, internalization was a significant predictor of drive for thinness; it accounted for $15 \%$ of the variance, $\beta=.38, t=4.63, p<.0001$. In the second equation, internalization was a significant predictor of self-objectification; it accounted for $24 \%$ of the variance, $\beta=.49, t=7.90, p<.0001$. In the third equation, the amount of variance accounted for by internalization reduced from $15 \%$ to $8 \%$, and self-objectification accounted for $5 \%$ of the variance independently. Overall, this mediational model accounted for $22 \%$ of the variance in drive for thinness, $F(2,207)=15.62, p<.0001$. Thus, selfobjectification partially mediated the relationship between internalization and drive for thinness, and internalization continued to contribute unique variance.

\section{Self-Objectification, Body Shame, and Drive for Thinness}

In the second analysis, a mediational model of eating disorders pathology was tested with selfobjectification, body shame, and drive for thinness. Figure 2 displays the results of a series of regression equations computed to test this model. In the first

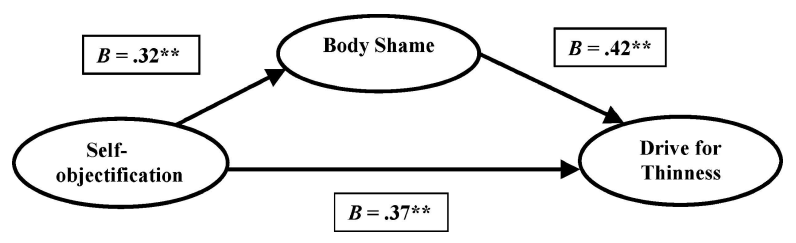

Fig. 2. Beta coefficients for the pathways among selfobjectification, body shame, and drive for thinness $\left({ }^{* *} p<.001\right)$. equation, self-objectification was a significant predictor of drive for thinness; it accounted for $14 \%$ of the variance, $\beta=.38, t=4.39, p<.0001$. In the second equation, self-objectification was a significant predictor of body shame; it accounted for $10 \%$ of the variance, $\beta=.32, t=4.87, p<.0001$. In the third equation, the amount of variance accounted for by self-objectification reduced from $14 \%$ to $7 \%$, whereas body shame accounted for $17 \%$ of the variance. Overall, the mediational model accounted for $30 \%$ of the variance in drive for thinness, $F(2,207)=$ $23.89, p<.0001$. Thus, body shame partially mediated the relationship between self-objectification and drive for thinness in women with eating disorders, and self-objectification continued to contribute unique variance.

\section{DISCUSSION}

The present effort contributes to objectification theory by providing empirical evidence for the link between self-objectification and drive for thinness in a clinical sample of women with eating disorders. First, internalized media ideals for appearance, but not information or pressures from media about appearance, contributed to self-objectification. This suggests that the viewing of sexually objectifying images of women in visual media (e.g., magazines, music videos, television shows) may be a contributing factor to the chronic viewing of oneself as a sexual object if those images become integrated into one's self-perception. The informational and pressuring aspects of the media have a minimal effect on further self-objectification once media messages have been made part of an individual's own belief system.

This finding has implications for the exposure to and experience of media by children and adolescents. Due to the correlational nature of the data, the causal relationship among these variables is unclear. It could be that internalized media ideals are contributing to a self-objectified perspective of the self. In this case, media literacy programs in which individuals are taught to evaluate a wide variety of media critically may significantly diminish current and future self-objectification (Steiner-Adair \& Vorenburg, 1999). However, it is possible that a self-objectified perspective on the self contributes to seeking out and internalizing media ideals for appearance. This perspective does not take as a given that, compared to men, women are just more susceptible to persuasion in advertising, and therefore more easily internalize 
media messages (see review, Martin, Gentry, \& Hill, 1999). Rather, this possibility raises the question: Why do adolescent girls and women appear to be motivated to use the media for the purposes of social, emotional, and physical identity? It also requires researchers to move beyond appearance concerns in their measurements of and explanations for internalizing media and the experience of self-objectification to avoid cyclical theoretical reasoning for these phenomena.

Second, internalized media ideals predicted drive for thinness directly, and also indirectly, through self-objectification. This mediational model suggests that in women with eating disorders, internalized media ideals for appearance may lead to a drive for thinness without necessarily requiring the experience of self-objectification. However, the model with self-objectification explained slightly more variance, which suggests that it does contribute to the development and/or maintenance of the drive for thinness in women with eating disorders. These results support the theoretical assumptions that visual media encourage women to objectify themselves and that both internalized media ideals and selfobjectification offer some account for eating disorders pathology.

Third, self-objectification predicted drive for thinness directly, and also indirectly, through body shame. This mediational model suggests that in women with eating disorders, self-objectification may lead to a drive for thinness without necessarily requiring the experience of body shame, which replicates previous tests of objectification theory in nonclinical samples (e.g., Fredrickson et al., 1998). However, the model with body shame explained substantially more variance, which suggests that it plays a significant role in the development and/or maintenance of a drive for thinness in women with eating disorders. The correlational nature of these two mediational models cannot establish conclusively the direction of effect, and thus causal links among these variables should be considered with caution. In sum, these findings suggest that self-objectification is present in women with eating disorders at admission to treatment; it negatively influences their emotional experiences, and it motivates the women to strive toward the attainment of unrealistic cultural body ideals.

As the length of hospitalization for women with eating disorders has significantly decreased over the past 15 years, it has become extremely important for inpatient programs to invest their time and energy optimally (Wiseman, Sunday, Klapper, Harris, \& Halmi, 2001). Treatment programs may further benefit patients when they are in a severe stage of their eating disorder (i.e., when they require inpatient treatment) by including opportunities to address the experience of sexual and self-objectification in women's lives. Because women are socialized to experience their efforts to monitor and improve their physical appearance as self-chosen or "natural," this way of relating to the self may not be experienced as egodystonic, uncomfortable, or unpleasant (Costanzo, 1992; Johnston, 1997). Consequently, most women who enter treatment are unlikely to identify selfobjectification as a problem because they are unaware that it is a potentially harmful, socially constructed form of self-perception. Certainly issues such as eating patterns, family dynamics, and individual psychological issues represent necessary therapeutic work. However, progress made in any of these areas may be undermined if socially constructed self-perceptions are not also targeted for change. When women continue to view themselves from a third person, rather than a first person, perspective, factors that contribute significantly to eating disorders pathology remain untouched.

In order to increase our understanding of the relationship between this kind of self-perception and recovery from eating disorders, further researchers should examine if and how self-objectification changes during treatment, and its specific effects on treatment outcomes including weight gain, symptomatic behavior, negative body image, self-esteem, interpersonal functioning, and time to relapse. In turn, this should point the way toward the development of treatment protocols for self-objectification and media literacy in both inpatient and outpatient settings. Prevention programs may further benefit from identifying and targeting the connections between self-perception, sociocultural influences, and eating behavior (e.g., GO GIRLS, 1999; Kater, 1998; Levine, Piran, \& Stoddard, 1999).

It is important to note that the results of the present study are constrained by the operational definitions underlying the questionnaires administered and the self-report nature of the data. Although the questionnaires utilized here have been well validated, other measures may be more appropriate to assess the experiences under investigation. In the present effort, Noll and Fredrickson's (1998) SOQ was utilized in order to compare the scores 
of this clinical sample to previously tested nonclinical samples. Also, this was the same measure of self-objectification used initially to test propositions of objectification theory.

Roberts (2004) provided a recent comparison of McKinley and Hyde's (1996) Objectified Body Consciousness (OBC)-Surveillance subscale to the SOQ. This comparison demonstrated that the SOQ specifically captures the experience of viewing the body from an outsider's perspective whereas the OBCSurveillance subscale includes items related to worry and concern about how the body appears to others. Thus, in the present sample of women who are particularly preoccupied with the body's outward appearance, the SOQ was considered an appropriate measure of this chronic, self-monitoring perspective of the self. However, the rank-order format of the scale has limitations, and one alternative measure of selfobjectification for future research is McKinley and Hyde's (1996) OBC-Surveillance scale.

The measure of eating disorder pathology was also narrow and limited to one subscale of the EDI2. Drive for thinness was used to capture the motivation for self-starvation in this population of women. Replication and/or efforts to extend this line of research might benefit from utilizing additional subscales of the EDI-2 or the Eating Disorders Examination (EDE; Fairburn \& Wilson, 1993), as well as nonappearance and nonweight focused measures.

Katzman and Lee (1997) asserted that "one cannot affect individual elective starvation without somehow treating the social world these individuals inhabit" (p. 392). Because many Western cultures sexually objectify the female body, all women to varying degrees are vulnerable to the experiences of self-objectification and disordered eating. It is imperative that we challenge these experiences at a social and cultural level and that we educate women about the negative consequences of selfobjectification early on in their physical, social, and emotional development. If children and adolescents can develop an empowered sense of self, they may be more likely to challenge power imbalances and the social construction of gender in ways that do not involve starvation and other abuses of their bodies (Katzman, Wooley, \& Fallon, 1994). Overall, the results of the present study suggest that socio-cultural explanations for the development and maintenance of eating disorder pathology should continue to be explored and integrated into the prevention and treatment efforts for clinical and nonclinical populations of women.

\section{REFERENCES}

American Psychiatric Association. (1994). Diagnostic and statistical manual of mental disorders (4th ed.). Washington, DC: Author.

Baron, R. M., \& Kenny, D. A. (1986). The moderator-mediator variable distinction in social psychological research: Conceptual, strategic, and statistical considerations. Journal of Personality and Social Psychology, 51, 1173-1182.

Bartky, S. L. (1990). Femininity and domination: Studies in the phenomenology of oppression. New York: Routledge.

Bruch, H. (1973). Eating disorders: Obesity, anorexia nervosa, and the person within. New York: Basic Books.

Bruch, H. (1982). Anorexia nervosa: Therapy and theory. American Journal of Psychiatry, 139, 1531-1538.

Calogero, R. M. (2004). A test of objectification theory: The effect of the male gaze on appearance concerns in college women. Psychology of Women Quarterly, 28, 16-21.

Calogero, R. M., Davis, W. N., \& Thompson, J. K. (2004). The sociocultural attitudes toward appearance questionnaire (SATAQ-3): Reliability and normative comparisons of eating disordered patients. Body Image, 1, 193-198.

Costanzo, P. R. (1992). External socialization and the development of adaptive individuation and social connection. In D. N. Ruble, P. R. Costanzo, \& M. E. Oliveri (Eds.), The social psychology of mental health (pp. 55-80). New York: Guilford.

Fairburn, C. G., \& Wilson, G. T. (1993). Binge eating: Nature, assessment, and treatment. New York: Guilford Press.

Fredrickson, B. L., \& Roberts, T. (1997). Objectification theory: Toward understanding women's lived experiences and mental health risks. Psychology of Women Quarterly, 21, 173206.

Fredrickson, B. L., Roberts, T.-A., Noll, S. M., Quinn, D. M., \& Twenge, J. M. (1998). That swimsuit becomes you: Sex differences in self-objectification, restrained eating, and math performance. Journal of Personality and Social Psychology, 75, 269-284.

Garner, D. M. (1991). EDI-2: Eating disorder inventory professional manual. Odessa, FL: Psychological Assessment Resources.

Garner, D. M., Olmstead, M. A., \& Polivy, J. (1983). Development and validation of a multidimensional eating disorder inventory for anorexia nervosa and bulimia. International Journal of Eating Disorders, 2, 15-34.

GO GIRLS: A curriculum for high school girls. (1999). Seattle, WA: Eating Disorders Awareness and Prevention.

Groesz, L. M., Levine, M. P., \& Murnen, S. K. (2002). The effect of experimental presentation of thin media images on body image satisfaction: A meta-analytic review. International Journal of Eating Disorders, 31, 1-16.

Johnston, J. (1997). Appearance obsession: Women's reactions to men's objectification of their bodies. In R. Levant \& G. Brooks (Eds.), Men and sex: New psychological perspectives (pp. 61-83). New York: Wiley.

Kaschak, E. (1992). Engendered lives: A new psychology of women's experience. New York: Basic Books.

Kater, K. J. (1998). Healthy body image: Teaching kids to eat and love their bodies too! Seattle, WA: Eating Disorders Awareness and Prevention.

Katzman, M. A., \& Lee, S. (1997). Beyond body image: The integration of feminist transcultural theories in the understanding of self-starvation. International Journal of Eating Disorders, $22,385-394$.

Katzman, M. A., Wooley, S. C., \& Fallon, P. (1994). Eating disorders: A gendered disorder. Eating Disorders Review, 5, 1-3.

Kim, J. L., \& Ward, L. M. (2004). Pleasure reading: Associations between young women's sexual attitudes and their reading 
of contemporary women's magazines. Psychology of Women Quarterly, 28, 48-58.

Levine, M. P., Piran, N., \& Stoddard, C. (1999). Mission more probable: Media literacy, activism, and advocacy as primary prevention. In N. Piran, M. P. Levine, \& C. SteinerAdair (Eds.), Preventing eating disorders: A handbook of interventions and special challenges (pp. 3-25). Philadelphia: Brunner/Mazel.

Martin, M. C., Gentry, J. W., \& Hill, R. P. (1999). The beauty myth and the persuasiveness of advertising: A look at adolescent girls and boys. In C. M. Macklin \& L. Carlson (Eds.), Advertising to children: Concepts and controversies (pp. 165-187). Thousand Oaks, CA: Sage Publications.

McKinley, N. M., \& Hyde, J. S. (1996). The objectified body consciousness scale. Psychology of Women Quarterly, 20, 181215.

Noll, S. M., \& Fredrickson, B. L. (1998). A mediational model linking self-objectification, body shame, and disordered eating. Psychology of Women Quarterly, 22, 623-636.

Roberts, T.-A. (2004). Female trouble: The menstrual selfevaluation scale and women's self-objectification. Psychology of Women Quarterly, 28, 22-26.

Slater, A., \& Tiggemann, M. (2002). A test of objectification theory in adolescent girls. Sex Roles, 46, 343-349.
Steiner-Adair, C., \& Vorenburg, A. P. (1999). Resisting weightism: Media literacy for elementary-school children. In N. Piran, M. P. Levine, \& C. Steiner-Adair (Eds.), Preventing eating disorders: A handbook of interventions and special challenges (pp. 105-121). Philadelphia: Brunner/Mazel.

Stice, E. (2001). Risk factors for eating disorder pathology: Recent advances and future directions. In R. H. Striegel-Moore \& L. Smolak (Eds.), Eating disorders: Innovative directions in research and practice (pp. 51-73). Washington, DC: American Psychological Association.

Thompson, J. K., van den Berg, P., Roehrig, M., Guarda, A. S., \& Heinberg, L. J. (2004). The sociocultural attitudes toward appearance scale-3 (SATAQ-3): Development and validation. International Journal of Eating Disorders, 35, 293-304.

Tiggeman, M., \& Lynch, J. (2001). Body image across the life span in adult women: The role of self-objectification. Developmental Psychology, 37, 243-253.

Tiggemann, M., \& Slater, A. (2001). A test of objectification theory in former dancers and non-dancers. Psychology of Women Quarterly, 25, 57-64.

Wiseman, C. V., Sunday, S. R., Klapper, F., Harris, W. A., \& Halmi, K. A. (2001). Changing patterns of hospitalization in eating disorder patients. International Journal of Eating Disorders, 30, 69-74. 